\title{
Utilização de métodos contraceptivos entre discentes do curso de Enfermagem de
} uma Universidade do Nordeste

\author{
Use of contraceptive methods among Nursing students from a University of the Northeast \\ Uso de métodos anticonceptivos entre estudiantes de Enfermería de la Universidad del Noreste
}

Recebido: 14/03/2021 | Revisado: 26/03/2021 | Aceito: 30/03/2021 | Publicado: 06/04/2021

Jéssica Soares dos Anjos Barboza

ORCID: https://orcid.org/0000-0001-6103-4456 Universidade Federal de Alagoas, Brasil

E-mail: jessicasdosab@gmail.com

Jovânia Marques de Oliveira e Silva

ORCID: https://orcid.org/0000-0001-7452-2651

Universidade Federal de Alagoas, Brasil E-mail: jovaniasilva@gmail.com

Sueli Teresinha Cruz Rodrigues ORCID: https://orcid.org/0000-0002-2697-4714

Universidade Federal de Alagoas, Brasil E-mail: suelitcr@gmail.com

Robert Lincoln Barros Melo

ORCID: https://orcid.org/0000-0002-3430-4411 Universidade Federal de Alagoas, Brasil E-mail:robert.lincoln.b@gmail.com

Roberta Santos Correia Silva

ORCID: https://orcid.org/0000-0002-6413-5460 Universidade Federal de Alagoas, Brasil E-mail: robertascorreias@gmail.com

Laryssa de Fátima Pereira Palmeira ORCID: https://orcid.org/0000-0002-4484-8682 Universidade Federal de Alagoas, Brasil E-mail: laryssa_palmeira_@hotmail.com

\begin{abstract}
Resumo
Este estudo busca analisar o perfil das acadêmicas do curso de Enfermagem quanto à utilização de métodos contraceptivos. Trata-se de estudo quantitativo descritivo de corte transversal e tem como participantes 133 alunas do curso, do $1^{\circ}$ ao $10^{\circ}$ período, ingressantes de 2013 a 2017. Os dados foram coletados de setembro a novembro de 2018 , através de entrevista semiestruturada. O perfil predominante das participantes é apresentado como: solteiras, com idade média de 23 anos, declarando-se estudantes. Delas, 94\% mantém relacionamento heterossexual, a sexarca com maior porcentagem foi de 16-20 anos (45\%), tendo o preservativo masculino como método contraceptivo mais utilizado. O coito interrompido (17\%) e o anticoncepcional oral (16\%) são os mais utilizados na sequência. Grande parcela das universitárias (84,6\%), afirmou nunca ter utilizado o preservativo feminino. Das participantes, $8 \%$ já obtiveram gravidez não planejada e 5\% já adquiriram alguma Infecção Sexualmente Transmissível (IST). Conclui-se que as estudantes, apesar de possuírem acesso facilitado à informação, ainda necessitam de espaços de educação em saúde sexual promovidos através de oferta de disciplinas eletivas como também rodas de conversas e oficinas relacionadas ao tema. Palavras-chave: Anticoncepção; Ensino; Estudantes de enfermagem; Gravidez não planejada; Infecção Sexualmente Transmissível.
\end{abstract}

\footnotetext{
Abstract

This study seeks to analyze the profile of nursing students under the use of contraceptive methods. This is a quantitative, descriptive, cross-sectional study, with 133 participants from the course, from the 1st to the 10th period, enrolled from 2013 to 2017. The data were collected from September to November 2018, through a semi-structured questionnaire. The predominant profile of the participants is presented as: single, with an average age of 23 years, declaring themselves students. Of these, 94\% maintain a heterosexual relationship, the sexarche with the highest percentage was 16-20 years old (45\%), with the male condom as the most used contraceptive method. Interrupted intercourse (17\%) and oral contraceptives (16\%) are the most used in the sequence. A large proportion of university students (84.6\%) said they had never used the female condom. Of the participants, $8 \%$ have already had unplanned pregnancies and $5 \%$ have already acquired some STI. It is concluded that the students, despite having easy access to information, still need spaces for sexual health education promoted through the provision of electives, as well as rounds of conversations and workshops related to the theme.
} 
Keywords: Contraception; Teaching; Nursing students; Unplanned pregnancy; Sexually Transmitted Infection.

\section{Resumen}

Este estudio busca analizar el perfil de estudiantes de enfermería bajo el uso de métodos anticonceptivos. Se trata de un estudio cuantitativo, descriptivo, transversal, con 133 participantes del curso, del $1^{\circ}$ al $10^{\circ}$ período, matriculados de 2013 a 2017. Los datos fueron recolectados de septiembre a noviembre de 2018, a través de un cuestionario semiestructurado. El perfil predominante de los participantes se presenta como: solteros, con una edad promedio de 23 años, declarándose estudiantes. De ellos, el 94\% tiene relación heterosexual, la sexarquia con mayor porcentaje fue la de 16 a 20 años (45\%), siendo el condón masculino el método anticonceptivo más utilizado. Las relaciones sexuales interrumpidas (17\%) y los anticonceptivos orales (16\%) son los más utilizados en la secuencia. Una gran proporción de estudiantes universitarios $(84,6 \%)$ dijo que nunca había usado el condón femenino. De las participantes, el $8 \%$ ya ha tenido embarazos no planificados y el 5\% ya ha adquirido alguna ITS. Se concluye que los estudiantes, a pesar de tener fácil acceso a la información, aún necesitan espacios de educación en salud sexual promovidos a través de la oferta de asignaturas optativas, así como rondas de conversaciones y talleres relacionados con la temática.

Palabras clave: Anticoncepción; Ensenãnza; Estudiantes de enfermería; Embarazo no planificado; Infección de Transmisión Sexual.

\section{Introdução}

A saúde da mulher, no Brasil, só veio a ser incorporada às políticas públicas nas primeiras décadas do século XX, e ainda assim, o enfoque do Ministério da Saúde residia no ciclo gravídico-puerperal, não assistindo a mulher em todas as suas dimensões. As questões sexuais e reprodutivas estavam restritas ao mundo particular. Em 1984, foi lançado o Programa de Assistência Integral à Saúde da Mulher (PAISM), que sofreu influência de movimentos feministas e constituiu-se de uma visão integral da saúde feminina, contemplando uma abordagem geracional, da adolescente à idosa, incluindo assim a atenção à saúde reprodutiva (Brasil, 2013; Brasil, 2016).

A saúde sexual e reprodutiva é definida como a liberdade de mulheres e homens desfrutarem de sua sexualidade, de forma segura e sem constrangimentos, tendo autonomia de decidir sobre quando e quantas vezes desejam se reproduzir, e em caso negativo, tendo direito de acesso à métodos eficazes e seguros para contracepção. Em 1996, através da Lei nº 9.263/96, que trata do planejamento familiar, estabelece-se que para exercer os direitos da população serão oferecidos todos os métodos de concepção e contracepção disponíveis que não coloquem em risco a vida e a saúde das pessoas, garantida a liberdade de opção (Brasil, 2013; Lago et al, 2020).

Em relatório divulgado pelo Departamento de Assuntos Econômicos e Sociais da Organização das Nações Unidas (ONU) intitulado Tendências do Uso de Métodos Anticoncepcionais no Mundo em 2015 estima-se que no Brasil, 79\% das mulheres em um relacionamento estável utilizam algum método contraceptivo para não engravidar e cerca de $86 \%$ dos brasileiros fazem planejamento reprodutivo. Os métodos mais utilizados para evitar a gravidez no país são a laqueadura (28\%), pílula anticoncepcional, preservativo e vasectomia, segundo o relatório (ONU News, 2016).

De acordo com a última Pesquisa Nacional de Demografia e Saúde (PNDS) realizada em 2006, verificou-se que as mulheres iniciavam a vida sexual cada vez mais cedo, e por consequência, a prática de anticoncepção também. A esterilização feminina $(25,9 \%)$ e a pílula oral $(27,4 \%)$ apareceram como sendo os dois métodos mais usados pelas mulheres. O uso de preservativo masculino cresceu em relação à pesquisa anterior e a contracepção de emergência aparece como quinto método mais usado entre todas as mulheres em idade reprodutiva (Brandão, 2019).

Iniciativas como a incorporação dos contraceptivos injetáveis na Relação Nacional de Medicamentos (RENAME), a ampliação da distribuição de contraceptivos através da atenção primária à saúde e a inclusão de anticoncepcionais hormonais orais (ACO) e injetáveis no programa Farmácia Popular do Brasil são estratégias usadas nos últimos anos para aumento das taxas de anticoncepção no país. Resoluções da Agência Nacional de Saúde Suplementar introduziram a obrigatoriedade da cobertura de procedimentos em contracepção como dispositivo intrauterino (DIU) e esterilizações (Olsen et al, 2018). 
Nesse sentido, acredita-se que a mais alta escolarização pode ter impactos diferenciados sobre a saúde sexual e reprodutiva da população. Na mulher, esse impacto relaciona-se ao adiamento de uniões conjugais, tendência ao maior uso de contraceptivos e menor chance de gestações não planejadas, devido ao maior acesso à informação pela ampliação dos meios digitais, crescente participação da mulher nos espaços públicos, inserção na Universidade e ampliação dos debates acerca das desigualdades nas relações de gênero (Bastos et al, 2008; Olsen et al, 2018).

No entanto, estudos mostram que apesar de os jovens mais escolarizados apresentarem maior chance de desfrutar de sua sexualidade com segurança, existem inconsistências no uso dos métodos contraceptivos de acordo com o envolvimento afetivo-amoroso. No relacionamento ocasional, existe a tendência de usar o preservativo masculino, direcionado a preocupação com ISTs. Já no namoro estável, cria-se uma confiança demasiada no parceiro, e o preservativo passa a ser substituído pela pílula anticoncepcional (Costa et al, 2017; Fontes et al., 2017).

Esse estudo justifica-se ao fato de a gravidez precoce e as ISTs serem problemas de saúde pública na atualidade, e o uso da dupla proteção, através do preservativo em associação a outro método contraceptivo, sua principal forma de prevenção. Além disso, a comunidade acadêmica, principalmente do curso de Enfermagem, tem grande potencial de escape dessa realidade, devido ao conhecimento adquirido em disciplinas do curso e na Universidade em si, levando a adoção de práticas sexuais mais saudáveis.

Apesar disso, é importante salientar que a discussão sobre temas relativos à sexualidade nos cursos de formação superior quase inexiste, realçando a lacuna nesse contexto educacional. Dessa forma, esse estudo tem como objetivo principal analisar o perfil das acadêmicas do curso de Enfermagem de uma Universidade do Nordeste quanto à utilização de métodos contraceptivos.

\section{Metodologia}

Trata-se de um estudo quantitativo descritivo de corte transversal, realizado em uma Universidade do Estado de Alagoas. As participantes foram acadêmicas do curso de Enfermagem, do $1^{\circ}$ ao $10^{\circ}$ período, ingressando na Universidade de 2013 a 2017 , do sexo feminino, que possuíssem idade igual ou superior a 18 anos, totalizando, ao final, 133 participantes, sendo que destas, 35 não possuíam vida sexual ativa.

Após aprovação do Comitê de Ética em Pesquisa, através do parecer n 2.857 .879 , os objetivos do estudo foram apresentados para as acadêmicas, que ao aceitarem participar, assinaram o TCLE e posteriormente responderam a entrevista. Todas as informações fornecidas pelas estudantes foram de sua livre escolha, sendo que para cada uma delas foi explicado o motivo da pesquisa e o conteúdo do instrumento de coleta de dados.

Foi elaborado um instrumento em forma de entrevista semiestruturada, que aborda as características sociodemográficas, os métodos contraceptivos utilizados, caracterização da vida sexual, a ocorrência de gravidez não planejada e ISTs e a coleta foi realizada entre os meses de setembro a novembro de 2018. Posteriormente foi realizada tabulação de dados e variáveis de estudo e as mesmas foram apresentadas em forma de tabelas e gráficos.

\section{Resultados e Discussão}

A Tabela 1 exibe resultados quanto às características sociodemográficas da população estudada: 
Tabela 1 - Características sociodemográficas das participantes.

\begin{tabular}{lll}
\hline Idade & Absoluta (N) & Relativa (\%) \\
\hline $18-23$ anos & 55 & 56 \\
$24-29$ anos & 37 & 38 \\
$30-35$ anos & 4 & 4 \\
Acima 35 anos & 2 & 2 \\
\hline Ocupação & Absoluta (N) & Relativa (\%) \\
\hline Estudante & 88 & 90 \\
Técnica em Enfermagem & 3 & 3 \\
Servidora pública & 3 & 3 \\
Professora & 2 & 2 \\
Agente Comum. de Saúde & 2 & 2 \\
\hline Estado Civil & Absoluta (N) & Relativa (\%) \\
\hline Solteira & 77 & 79 \\
Casada & 15 & 15 \\
Outro & 6 & 6 \\
\hline
\end{tabular}

Fonte: Autores (2021).

Quanto às características sociodemográficas das participantes, a Tabela 1 evidencia que a faixa etária com maior concentração é a dos 18-23 anos (56\%), tendo como idade média 23 anos, declarando sua ocupação como estudante (90\%), além de serem majoritariamente solteiras (79\%). A partir dos resultados obtidos observamos que as participantes da pesquisa ingressam jovens na Universidade, além de estarem em plena idade fértil e reprodutiva. Sobre a faixa etária, Castro et al (2016) afirma que as IST são "mais prevalentes entre jovens de 14 a 29 anos e os universitários constituem população altamente exposta", corroborando com o dado da pesquisa.

Gráfico 1 - Distribuição das estudantes por tipo de relacionamento sexual.

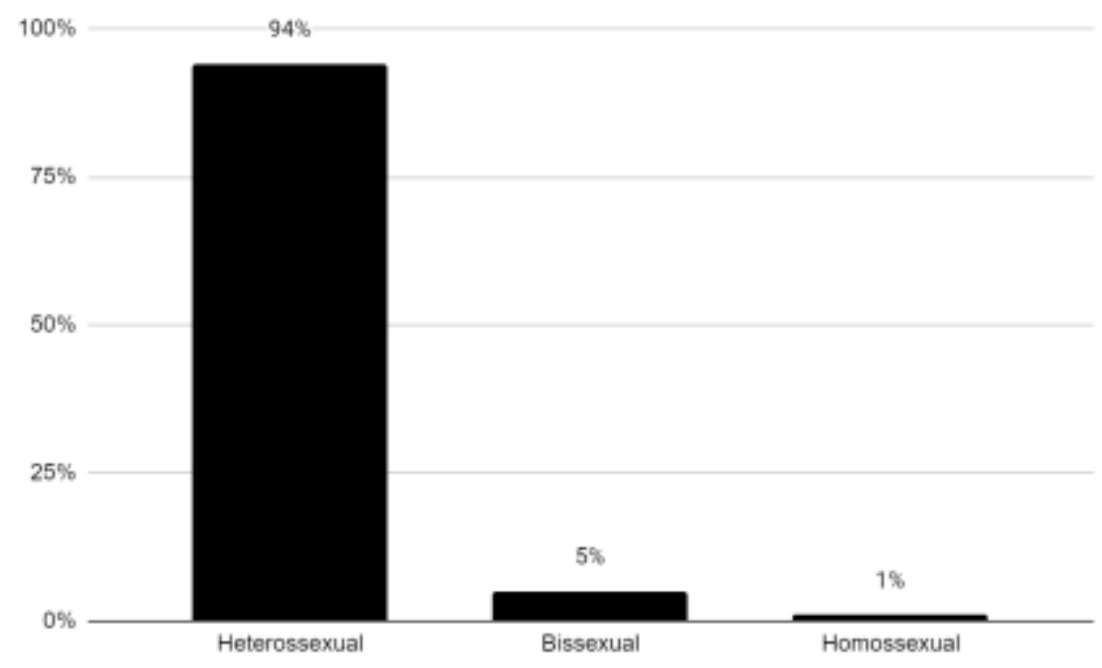

Fonte: Autores (2021). 
Em relação ao tipo de relacionamento sexual, $5 \%(\mathrm{n}=5)$ das estudantes informaram a bissexualidade, $1 \%(\mathrm{n}=1)$ informou a homossexualidade, enquanto $94 \%$ (n=92) informaram a heterossexualidade, conforme pode-se observar no Gráfico 1.

Pesquisas realizadas com mulheres lésbicas no Brasil indicam que estas tendem a procurar os serviços de saúde com menor frequência do que as pacientes heterossexuais. Ainda hoje, existem dificuldades e silenciamentos ligados à sexualidade e principalmente sobre a homossexualidade feminina, que se reflete nos cuidados e no acesso em relação à saúde dessa população. As mulheres homossexuais ou bissexuais devem ser orientadas quanto a comportamentos e práticas sexuais de risco, como múltiplas parcerias, uso inconsistente de preservativo em relações sexuais com homens, sexo com parceira menstruada e compartilhamento de acessórios sexuais (Carvalho et al, 2013; Fernandes et al, 2018).

Gráfico 2 - Distribuição das estudantes quanto à sexarca.

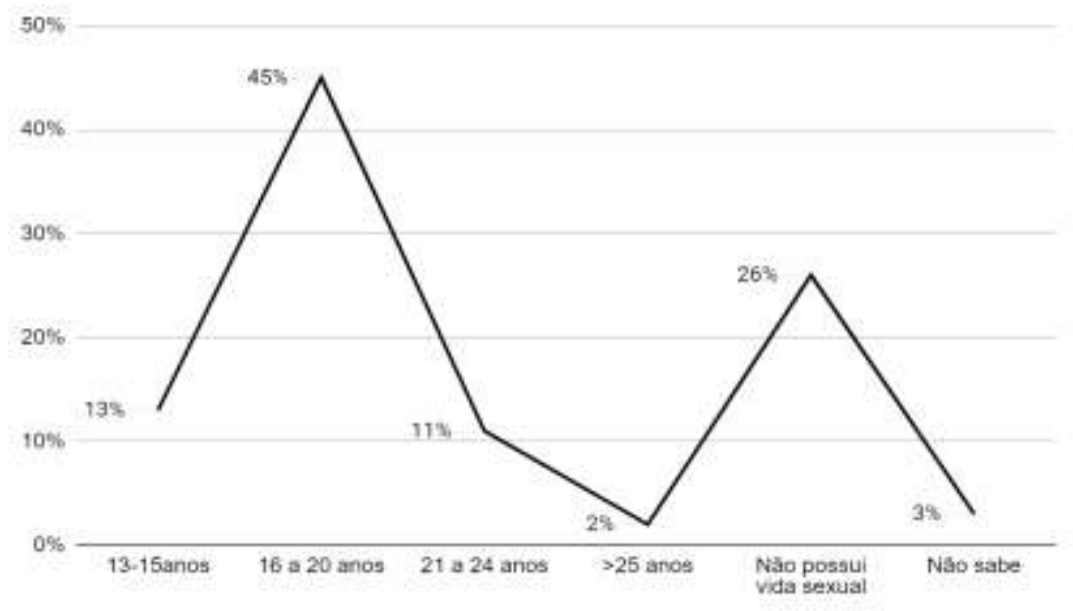

Fonte: Autores (2021).

O Gráfico 2 traz a distribuição das estudantes quanto ao momento de sua primeira relação sexual, ou sexarca. Verificase que a faixa etária com maior concentração é a dos 16-20 anos $(45 \%, \mathrm{n}=60)$. Esse dado corrobora com o estudo realizado por Costa et al (2017), onde verificou-se que as universitárias iniciaram sua vida sexual no período próximo ao ingresso ou durante a universidade, dado que estes dois eventos representam marcos em direção a autonomia e independências próprias da vida adulta.

Apesar disso, também chama a atenção o fato de $13 \%$ das participantes terem iniciado a vida sexual na faixa dos 13-15 anos. Quanto a isso, Brasil (2013) afirma que "as mulheres estão começando sua atividade sexual cada vez mais cedo, o mesmo sucedendo com a prática da anticoncepção". Em 2006, segundo a PNDS, até os 15 anos, 33\% das mulheres já haviam tido relações sexuais.

Em um estudo realizado por Roteli-Martins et al (2007) envolvendo mulheres usuárias do Sistema Único de Saúde (SUS) das cidades de São Paulo, Campinas e Porto Alegre verificou-se que aproximadamente 20\% das mulheres relataram sexarca com idade igual ou menor a 15 anos, sendo que a maioria referiu o início da atividade sexual entre 14 e 20 anos (76,4\%), corroborando com os dados dessa pesquisa.

Outro dado que chama atenção neste gráfico é o fato de $26 \%(\mathrm{n}=35)$ das estudantes ainda não terem iniciado a vida sexual, apesar de estarem na faixa etária de maior prevalência da mesma e de maior interesse sexual. Nesse sentido, acreditamos 
que esse grupo de mulheres também deve ser alvo de ações de prevenção e promoção da saúde sexual e reprodutiva, a fim de conhecer os métodos, reconhecer sua importância e mais tarde usá-los de forma coerente.

Gráfico 3 - Distribuição das estudantes quanto ao(s) método(s) contraceptivo(s) utilizado(s) atualmente.

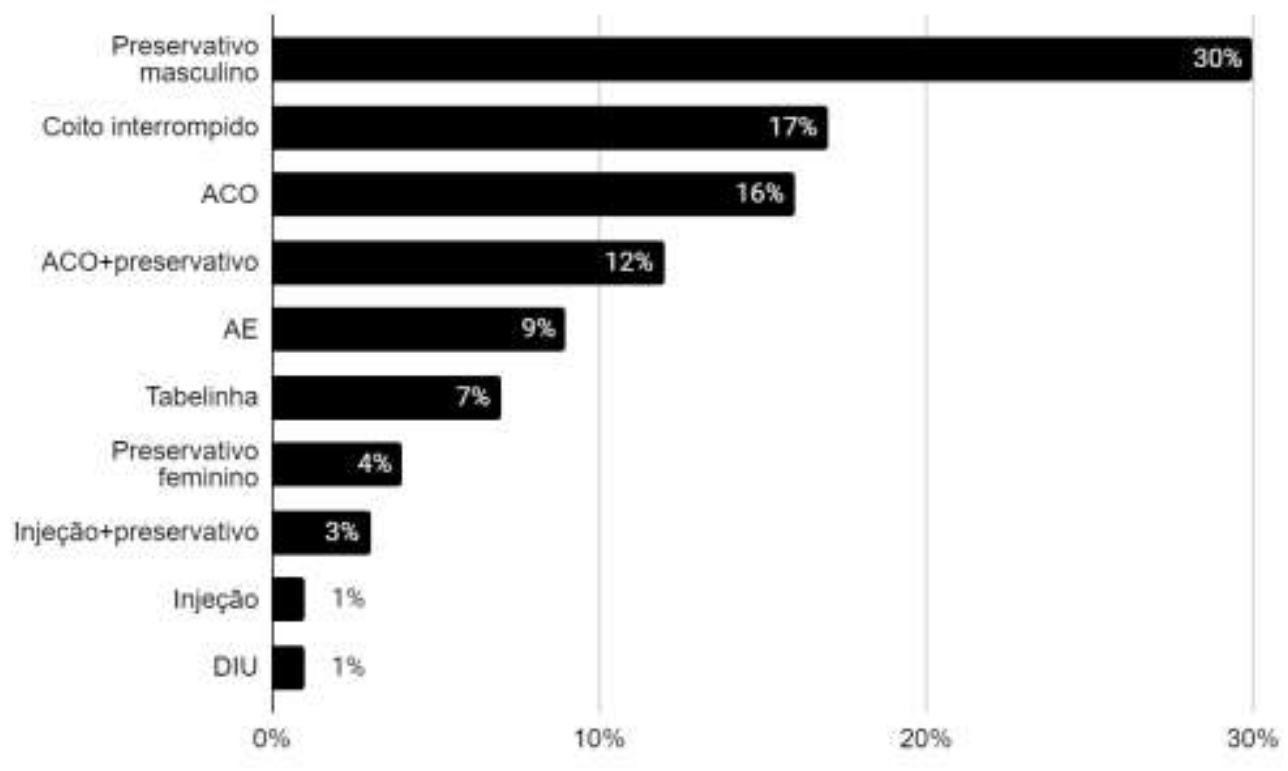

Fonte: Autores (2021).

Quanto ao método contraceptivo utilizado atualmente pelas estudantes, o Gráfico 4 evidencia o uso do preservativo masculino (30\%) por sua maior parte, correspondendo a outros trabalhos parecidos, envolvendo tanto adolescentes, quanto estudantes da área de saúde, em que o método contraconceptivo mais utilizado também foi o preservativo masculino, por ser um método mais acessível. Estudos indicam ainda que existe uma tendência ao abandono do preservativo à medida que as relações afetivas tornam-se mais estreitas (Costa et al, 2017).

Ainda no Gráfico 3 podemos observar que o uso do preservativo masculino é seguido do coito interrompido (17\%) e Anticoncepcional Oral (ACO) (16\%). O uso concomitante do ACO+preservativo (12\%) ou Injeção+preservativo (3\%), que se configura como dupla proteção, ainda atinge pequena parcela das universitárias.

A Anticoncepção de Emergência (AE) já foi utilizada por cerca de $9 \%$ das estudantes, que afirmaram tê-la usado "raramente", duas vezes em toda a vida sexual", "1 ou 2 vezes por ano" e "quando não uso camisinha". Este dado torna-se interessante ao notarmos que apesar das estudantes possuírem amplo acesso e conhecimento a métodos contraceptivos de alta eficácia, optaram pelo uso de $\mathrm{AE}$, que é um método contraceptivo que deve ser utilizado com cautela, visto que o uso rotineiro compromete sua eficácia (Costa et al, 2017).

Chama a atenção a alta utilização da prática de coito interrompido (17\%), estando à frente até mesmo do anticoncepcional oral. Apesar de muito visto na literatura, o mesmo é desaconselhado pela OMS e pelo Ministério da Saúde (MS) que afirmam que esta prática pode levar a alto índice de falha e gravidez não planejada pois as secreções liberadas pelo pênis na fase de excitação podem conter espermatozoides, além de não proteger contra ISTs. Esse dado difere da literatura nacional, que aponta que contraceptivos hormonais aparecem como o segundo método contraceptivo mais utilizado entre graduandos de universidades do Brasil (Brasil, 2018; Moreira, 2018).

O uso da tabelinha, outro método comportamental, é utilizado por $7 \%$ das entrevistadas e justificado pelo grau de conhecimento que a estudante tem pelo seu ciclo menstrual. A injeção anticoncepcional (1\%) e o DIU (1\%) são os métodos 
menos utilizados. Apenas uma estudante faz uso do DIU de cobre e justifica seu uso por ser "um método seguro, não hormonal e efetivo". Olsen (2018) afirma que embora o DIU de cobre seja ofertado gratuitamente no SUS, é poucas vezes oferecido às mulheres, particularmente às que não tiveram filhos.

Conhecidos os principais métodos contraceptivos utilizados pelas estudantes, a Tabela 2 tem o objetivo de distribuí-las quanto aos motivos para a escolha dos mesmos.

Tabela 2 - Distribuição das estudantes segundo motivo pelo qual escolheu o método contraceptivo utilizado atualmente.

\begin{tabular}{ccc}
\hline Motivo da escolha & Absoluto (N) & Relativo (\%) \\
\hline Evitar gravidez & 20 & 20,4 \\
Segurança & 17 & 17,3 \\
Praticidade para usar & 14 & 14,2 \\
Facilidade para obter & 13 & 13,2 \\
Evitar efeitos colaterais hormonais & 8 & 8,1 \\
Hábito/ Comodidade & 6 & 6,1 \\
Evitar gravidez e ISTs & 5 & 5,1 \\
Indicação médica & 4 & 4 \\
Por conhecer bem o ciclo menstrual & 2 & 2 \\
Por ter acordado com o parceiro & 1 & 1 \\
Amamentação & 1 & 1 \\
Não respondeu & 9 & 9,1 \\
& & \\
\hline
\end{tabular}

Fonte: Autores (2021).

Para 20,4\% das estudantes, o motivo de escolha do método contraceptivo utilizado atualmente é "evitar gravidez", seguida da segurança oferecida pelo método (17,3\%), sua praticidade para usar $(14,2 \%)$ e facilidade para obter $(13,2 \%)$. Podemos observar então que a maior preocupação das estudantes quanto ao uso de métodos contraceptivos é evitar uma gravidez não planejada, e a prevenção quanto ISTs só é relatada por 5,1\% das participantes. Esse dado se iguala a estudo realizado por Delatorre (2015) que identificou que "o uso do preservativo entre as jovens aparece muito mais relacionado à prevenção da gravidez do que para evitar a aquisição de uma DST".

Ainda com relação à praticidade, estudo realizado com universitários, por Pirotta et al (2002), evidenciou que os entrevistados consideravam o preservativo masculino um método prático e fácil de usar. Leite et al (2007) argumenta que a preferência pelo uso do preservativo em relação a outros métodos parece estar associada à praticidade, ao baixo custo financeiro e à ausência de efeitos colaterais.

Com relação à facilidade de acesso, Olsen et al (2018) afirma que o acesso à maior variabilidade de métodos favorece a liberdade de escolha possibilitando maiores chances de prevenção de gestações não planejadas. Segundo os autores outros países têm investido nessa ampliação: na Colômbia, o implante subdérmico é fornecido gratuitamente à população desde 2008; no México, os implantes, o adesivo e o DIU com levonorgestrel são disponibilizados gratuitamente; na Inglaterra, há 15 tipos de métodos contraceptivos fornecidos gratuitamente pelo serviço de saúde nacional, incluindo os anéis vaginais e adesivos. 
Tabela 3 - Distribuição das estudantes segundo utilização/não utilização do preservativo feminino e os motivos para tal decisão.

\begin{tabular}{lcc}
\hline Utilização do preservativo feminino & Absoluto (N) & Relativo (\%) \\
\hline Sim & 15 & 15,4 \\
Não & 83 & 84,6 \\
\hline Motivos relacionados à não utilização & Absoluto (N) & Relativo (\%) \\
\hline Parece desconfortável & 23 & 23,4 \\
Parece incômodo & 19 & 19,3 \\
Menos acessível & 9 & 9,1 \\
Nunca precisou & 2 & 2 \\
Não tem vontade & 1 & 1 \\
Não sabe usar & 1 & 1 \\
Não gosta do formato & 1 & 1 \\
Difícil de colocar & 1 & 1 \\
Não justificou & 29 & 29,5 \\
\hline Motivos relacionados à utilização & Absoluto (N) & Relativo (\%) \\
\hline Curiosidade & 9 & 9,1 \\
O parceiro não quis usar o masculino & 1 & 1 \\
Maior conforto e prazer & 1 & 1 \\
Gosto de usar & 1 & \\
& & \\
\hline
\end{tabular}

Fonte: Autores (2021).

A Tabela 3 tem o objetivo de quantificar o número de estudantes que já utilizaram o preservativo feminino e os motivos para tal. Mais da metade das participantes $(84,6 \%)$ afirmaram nunca ter utilizado este método, e uma pequena parcela (15,3\%) afirma já ter utilizado o preservativo feminino. Dentre os motivos mais citados para a não utilização do método, estão o fato de "parecer desconfortável" e parecer incômodo", ou seja, as estudantes nunca utilizaram o mesmo e baseiam sua justificativa num pré-conceito ou no que já ouviram falar.

É importante salientar que este preservativo proporciona a autonomia feminina, pois oferece um meio de prevenção à mulher que independe do homem para sua colocação e utilização, é confortável e seguro, possuindo menos chances de rompimento em comparação ao preservativo masculino (Oliveira et al, 2010).

Pernas (2014) afirma que no Brasil, em comparação aos países hispânicos, existem elevadas taxas de adesão, continuidade e aceitabilidade quanto ao preservativo feminino ao passo que as mulheres têm domínio sobre a forma correta como usá-lo. Neste estudo, ao contrário dos dados encontrados na literatura, as estudantes afirmaram ter dificuldade e não saber usar o preservativo feminino. No estudo de Moreira et al (2018) a disponibilidade do preservativo feminino também tem sido apontada como importante para que ocorra o uso. 
Gráfico 4 - Distribuição das estudantes quanto a ocorrência de gravidez não planejada e ISTs.

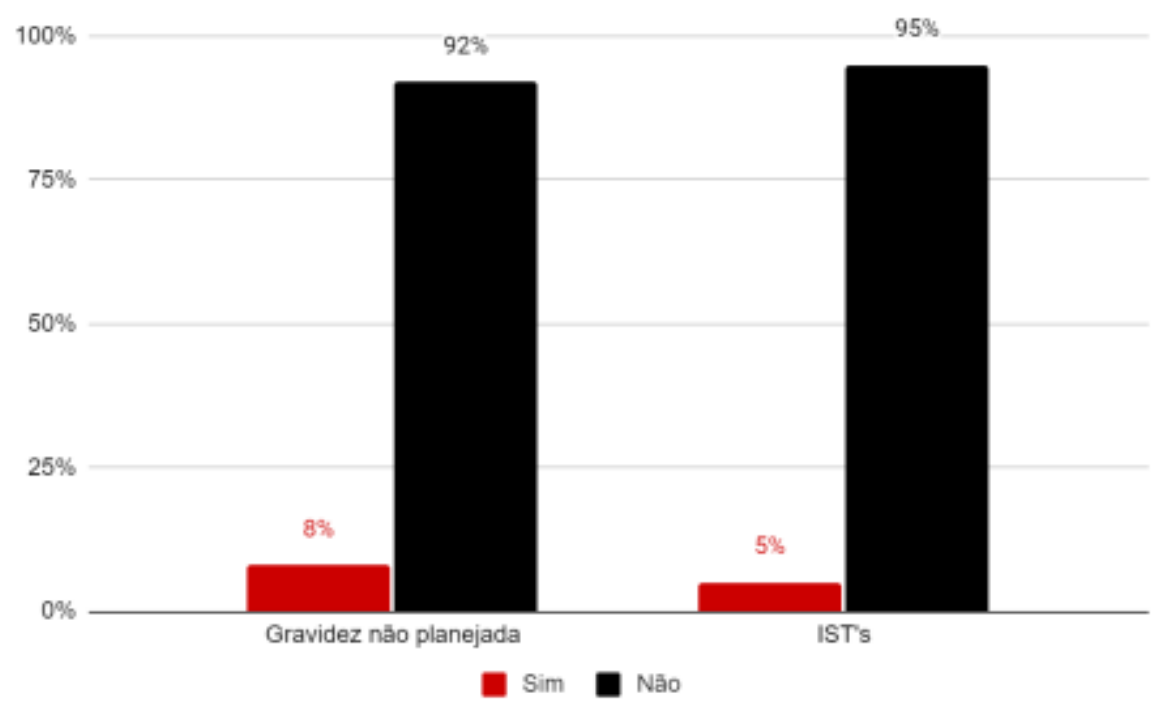

Fonte: Autores (2021).

Como é apresentado no Gráfico 4 o grupo pesquisado apresenta baixa fecundidade. Entre as universitárias que haviam iniciado a vida sexual, $8 \%$ referiram uma ou mais gestações não planejadas, e dentre estas, $4 \%$ já estavam cursando a graduação ao engravidar. Quanto às ISTs, 5\% das estudantes referiram já ter adquirido alguma infecção, dentre elas a Candidíase, Papilomavírus Humano (HPV) e Clamídia. A partir desses dados, consegue-se visualizar que apesar de as estudantes declararam fazer uso de métodos contraceptivos, existem inconsistências, culminando na ocorrência da gravidez não planejada e ISTs.

Segundo Fonte et al (2018) estudos apontam a vulnerabilidade da população de jovens universitários às ISTs, tendo em vista a variabilidade de parceiros e o início da vida sexual. O cenário universitário favorece o aparecimento e consolidação de determinados comportamentos, especialmente, relacionados ao consumo de álcool e outras drogas e de encontros sexuais. Visto isso, concordamos com os autores quando os mesmos afirmam que a articulação entre a universidade e os serviços de saúde ainda é precária e devem ser pensadas estratégias nessa perspectiva.

Pereira e Silva et al (2014) apontam que estudos realizados com acadêmicos da área da saúde demonstram que seus conhecimentos sobre ISTs, HIV/Aids e métodos contraceptivos não são suficientes para reduzir a exposição a práticas sexuais de risco. Nesse sentido, consideramos vital que em estudos futuros seja pesquisado sobre o nível de conhecimento das universitárias quanto às ISTs, visto que a candidíase se trata de uma infecção fúngica e foi apontada como IST pelas mesmas.

Em relação à gestação não planejada, Gemaque e Souza (2016) consideram que se trata de um dos maiores motivos de evasão universitária feminina, acompanhado do casamento e nascimento dos filhos e nesse sentido as Instituições devem pensar em estratégias para combatê-la, através de ações concretas para retenção do aluno.

\section{Considerações Finais}

Diante de todo o exposto, considera-se que os objetivos propostos para o estudo puderam ser alcançados e o perfil das estudantes quanto ao uso dos métodos contraceptivos pôde ser traçado, ainda que ele seja passível de alterações, visto que os comportamentos sexuais estão em constante mudança. É necessário que o grande número de mulheres que ingressam todos os anos no curso de Enfermagem se aproprie de todo o conhecimento que a Universidade oferece, tornando-se mulheres e profissionais detentoras do conhecimento dos direitos sexuais e reprodutivos. 
Durante o estudo foi possível identificar que o perfil das estudantes se assemelha em muitos aspectos com outros estudos realizados pelo país, mas devemos atentar-nos principalmente ao alto uso da prática do coito interrompido, que é desaconselhada por entidades nacionais e internacionais. Observou-se ainda que é necessário dar mais importância às ISTs, visto que a maior parte das universitárias se preocupa apenas com a gravidez não planejada ao fazer uso de determinado método, apresentando postura vulnerável.

Sendo assim, conclui-se que as estudantes, apesar de possuírem acesso facilitado à informação, ainda necessitam de espaços de educação em saúde sexual, sejam eles facilitados pelo Centro Acadêmico, Ligas, disciplina de Saúde da Mulher ou pela própria coordenação, como através de oferta de disciplina eletiva, rodas de conversa e oficinas sobre o uso correto do preservativo, parcerias com a Secretaria Municipal de Saúde em eventos e reuniões de grupos de pesquisa, a fim de tratar esse assunto de forma impessoal e não apenas como conteúdo acadêmico.

É importante ainda que os serviços de saúde considerem as universidades como um espaço de articulação intersetorial para promover uma mobilização social de enfrentamento às IST, bem como de outros agravos que acometem a população jovem. Enquanto integrantes da sociedade, temos o dever de garantir os direitos fundamentais da pessoa humana e isso inclui os direitos sexuais e reprodutivos.

Consideramos que os resultados obtidos permitem adquirir um conhecimento mais real sobre os comportamentos sexuais que podem colocar em risco os estudantes universitários, além de contribuir na promoção e prevenção da saúde sexual, já que essas informações poderão ajudar na intervenção pedagógica focada para esse grupo e assim promover comportamentos sexuais mais saudáveis. Por fim, esperamos que o estudo contribua como incentivo para futuras pesquisas na área da saúde sexual e que muitas mulheres e estudantes venham a ser beneficiadas por eles.

\section{Referências}

Bastos, M. R., et al. (2008). Práticas contraceptivas entre jovens universitárias: o uso da anticoncepção de emergência. Texto \& Contexto - Enfermagem, 17(3), 447-456. https://doi.org/10.1590/S0104-07072008000300005

Brandão, E. R. (2019). Métodos contraceptivos reversíveis de longa duração no Sistema Único de Saúde: o debate sobre a (in)disciplina da mulher. Ciênc. saúde colet. 24 (3). https://doi.org/10.1590/1413-81232018243.10932017.

Brasil, M. S. (2013). Secretaria de Atenção à Saúde. Departamento de Atenção Básica. Saúde sexual e saúde reprodutiva / Ministério da Saúde, Secretaria de Atenção à Saúde, Departamento de Atenção Básica. Ministério da Saúde.

Brasil. MS. (2016). Protocolos da Atenção Básica: Saúde das Mulheres / Ministério da Saúde, Instituto Sírio-Libanês de Ensino e Pesquisa - Brasília: Ministério da Saúde.

Brasil. MS. (2018). Secretaria de Atenção à Saúde. Departamento de Ações Programáticas Estratégicas. Saúde Sexual e Saúde Reprodutiva os homens como sujeitos de cuidado / Ministério da Saúde, Secretaria de Atenção à Saúde, Departamento de Ações Programáticas Estratégicas. - Brasília: Ministério da Saúde.

Carvalho, C. S., et al. (2013). O dispositivo "saúde de mulheres lésbicas": (in)visibilidade e direitos. Revista Psicologia Política, 13 (26), 111-127.

Castro, E. L., et al. (2016). O conhecimento e o ensino sobre doenças sexualmente transmissíveis entre universitários. Ciência \& Saúde Coletiva.

Costa, A. G. S., et al. (2017). Práticas Contraceptivas Entre Universitárias Da Faculdade De Medicina De Valença - RJ. Brazilian Journal of Surgery and Clinical Research. 19(1), 64-70.

Delatorre, M. Z., et al. (2015). Conhecimentos e práticas sobre métodos contraceptivos em estudantes universitários. Rev. SPAGESP: Ribeirão Preto.

Fernandes, M., et al. (2018). Saúde das mulheres lésbicas e atenção à saúde: nem integralidade, nem equidade diante das invisibilidades. Diversidade sexual e de gênero.

Fonte, V. R. F. da, et al. (2018). Jovens universitários e o conhecimento acerca das infecções sexualmente transmissíveis. Escola Anna Nery, 22 (2), e20170318. https://doi.org/10.1590/2177-9465-ean-2017-0318

Fontes, M. B., et al. (2017). Fatores determinantes de conhecimentos, atitudes e práticas em DST/Aids e hepatites virais, entre jovens de 18 a 29 anos, no Brasil. Ciência \& Saúde Coletiva, 22(4), 1343-1352. https://dx.doi.org/10.1590/1413-81232017224.12852015

Gemaque, L. S. B.\& Souza, L. G. (2016). Diplomação, Retenção E Evasão: estudo com enfoque na evasão dos cursos de graduação na Universidade Federal do Maranhão no período de 2008 a 2010. Ensino \& Multidisciplinaridade, 2(1), 84-105. 
Research, Society and Development, v. 10, n. 4, e20410413886, 2021

(CC BY 4.0) | ISSN 2525-3409 | DOI: http://dx.doi.org/10.33448/rsd-v10i4.13886

Lago, T.G., et al. (2020). Diferenciais da prática contraceptiva no Município de São Paulo, Brasil: resultados do inquérito populacional Ouvindo Mulheres. Cad. Saúde Pública 36 (10). https://doi.org/10.1590/0102-311X00096919

Leite F. et al. (2007). Saber e prática contraceptiva e prevenção de DST/HIV/AIDS em universitários da área da saúde. Rev. bras. enferm.

Moreira, L.R., et al. (2018). Uso de preservativos na última relação sexual entre universitários: quantos usam e quem são? Ciência \& Saúde Coletiva. 23(4), 1255-1266. https://doi.org/10.1590/1413-81232018234.16492016

Oliveira, J. C. P., et al. (2010). O conhecimento das mulheres sobre o uso do preservativo feminino. Ágora: R. Divulg. Cient., 7(1).

Olsen, J.bM., et al. (2018). Práticas contraceptivas de mulheres jovens: inquérito domiciliar no Município de São Paulo, Brasil. Cad. Saúde Pública. DOI: $10.1590 / 0102-311$ X00019617

Onu News (2016). 64\% das mulheres usaram algum tipo de método anticoncepcional em 2015. https://news.un.org/pt/story/2016/01/1538811-64-das-mulheresusaram-algum-tipo-de-metodo-anticoncepcional-em-2015

Pereira e Silva, L. et al. (2014). Comportamento sexual dos acadêmicos ingressantes em cursos da área da saúde de uma universidade pública. Revista de Enfermagem e Atenção à Saúde. REAS [Internet];3(1):39-52

Pernas, C. S. B. (2014) Conhecimento, atitude e prevalência do Preservativo Feminino nos jovens adultos portugueses. Dissertação de Mestrado. Universidade do Algarve.

Pirotta, K. C., et al. (2002). Juventude e Saúde Reprodutiva: valores e condutas relacionados com a contracepção entre universitários. Associação Brasileira de Estudos Populacionais.

Roteli-Martins, C. M., et al. (2007). Associação entre idade ao início da atividade sexual e subseqüente infecção por papilomavírus humano: resultados de um programa de rastreamento brasileiro. Revista Brasileira de Ginecologia e Obstetrícia, 29(11), 580-587. 